\title{
Central Bank Independence and Price Stability: Evidence from 23 OECD-countries*
}

\author{
Sven-Olov Daunfeldt ${ }^{\dagger}$ and Xavier de Luna \\ The Swedish Research Institute of Trade (HUI). \\ Department of Statistics, Umeå University.
}

June 6, 2003

\begin{abstract}
In this paper, we use a non-parametric regression method to compare the transition process from high to low inflation with the implementation dates of central bank independence reforms. In a majority of the countries, price stability is achieved before more independence is given to the central bank. This suggests that the credibility of a low inflation goal can be achieved without institutional reforms which grant the central bank more independence from the political policymakers.
\end{abstract}

Key words: Central bank reform; inflation targeting; monetary policy; non-parametric decomposition

JEL classification: C14; E52; E58

\footnotetext{
${ }^{*}$ We would like to thank Thomas Jonsson for providing us with announcement and implementation dates for central bank independence reforms. Financial support from the Swedish Foundation for International Cooperation in Research and Higher education (STINT) is gratefully acknowledged.

${ }^{\dagger}$ The Swedish Research Institute of Trade (HUI), S-103 29, Stockholm, Sweden. Tel: +46 (0)8 76272 80; Fax: +46 (0)8 67976 06; E-mail: so.daunfeldt@econ.umu.se
} 


\section{Introduction}

Over the last two decades a majority of the OECD countries have implemented institutional reforms which grant their central banks more independence from the political process. Kydland and Prescott's (1977) and Barro and Gordon's (1983) work on time inconsistency in monetary policy, together with Rogoff's (1985) suggestion that a central bank with more inflation averse preferences can make a low inflation policy credible, constitute the theoretical rationale for these central bank reforms.

Empirical support for central bank independence, to cite a few examples, has been provided by Grilli et al. (1991), Cukierman et al. (1992), Alesina and Summers (1993), Jonsson (1995), and Eijffinger et al. (1998). Using an index reflecting the degree of central bank independence, these studies found a negative correlation between central bank independence and average inflation. Alesina and Summers (1993) could not find any correlation between central bank independence and other real economic variables such as unemployment, real economic growth, and real interest rates. As a result, there is a broad consensus that central bank independence improves the likelihood of achieving a low inflation goal at no real economic costs.

However, this view has recently been subject to both theoretical and empirical criticism. ${ }^{1}$ For instance, the models by Kydland and Prescott (1977) and Barro and Gordon (1983) does not consider fiscal policy. As suggested by Agell, Calmfors and Jonsson (1996) and Blake and Weale (1998), even though an independent central bank implement the monetary policy, a low

\footnotetext{
${ }^{1}$ For an overview, see Forder (1998a) and Hayo and Hefeker (2002).
} 
inflation policy may lack credibility because policymakers still have an incentive to create surprise inflation through an expansive fiscal policy. It is also commonly argued (see e.g., Posen, 1993; Forder, 1996; Hayo, 1998) that central bank independence and the commitment to a low inflation rule is jointly determined by social attitudes, i.e., central bank independence is an endogenously determined variable. This suggests that independent central banks are successful in implementing low and stable inflation merely because their independence reflects a social attitude that supports low inflation. Forder (1998b) has also shown that a plausible correction of the measure of central bank independence applied in previous empirical work leads to results that show no correlation between central bank independence and average inflation. Moreover, Mangano (1998) shows that the most commonly used indices of central bank independence are subject to a rather large subjectivity bias. Hence, despite the broad consensus that central bank independence improves the credibility of a pre-commited low inflation policy, the empirical studies that support more independence for the central banks from the political process are not uncontroversial.

Since the empirical studies of Grilli et al. (1991), Cukierman et al. (1992), Alesina and Summer (1993) and Jonsson (1995), among others, efforts to evaluate central bank independence from a different methodological perspective have been scarce. One exception is Posen (1998) who studies the relationship between central bank independence and the cost of disinflation. According to Posen (1998), countries with more independent central banks should have higher credibility and therefore lower cost of disinflation measured as the sacrifice ratio. On the contrary to this prediction, his results 
show a positive and significant relation between central bank independence and the cost of disinflation. However, since Posen (1998) uses the same index for central bank independence as was used in previous empirical work, his study is also subject to the same criticism.

The implementation dates of central bank reforms constitute a neglected source of information for investigating how central bank independence affects the credibility of a pre-commited goal of low inflation. The purpose of this paper is to study how institutional reforms granting the central bank more independence from the political policymakers have affected the efforts to achieve low and stable inflation in 23 OECD-countries. Because the reforms have by definition increased the degree of independence of the central banks, our analysis does not suffer from the subjectivity problem that characterizes the construction of independence indexes.

A non-parametric decomposition of the inflation series in three frequencies of variation components is obtained with locally weighted regression (see Cleveland, Devlin and Grosse, 1988; Cleveland et al., 1990). This allows us to separate out the long term inflation component, the main object of interest, from medium term cyclical and transient variation as well as short term shocks and irregularities. The long term inflation component is then compared to the increase in independence of the central bank, to investigate whether this reform is responsible for the shift from high to low inflation observed in most of the countries covered in the study. No such effect is evident from the analysis, which suggests that central bank independence is not necessary in order to achieve low and stable inflation. The results show, moreover, that the transition from high to low inflation cannot be attributed 
to the more extensive use of inflation targeting during the 1990s.

The article is organized as follows: the next section describes the empirical study, results are presented and commented on in Section 3, while Section 4 contains our main conclusions.

\section{Decomposition of the inflation}

We have based our study on the quarterly consumer price index (CPI) published by the OECD. We define the inflation at time $t, \pi_{t}$, as

$$
\pi_{t}=\left(\mathrm{CPI}_{t}-\mathrm{CPI}_{t-4}\right) / \mathrm{CPI}_{t-4},
$$

i.e., the relative increase (or decrease) in prices within one year interval. This quarterly measured annual inflation should not show seasonal variation and this is confirmed in the analysis performed below. We examined inflation starting in 1975 , because our interest lies in the shift from high inflation following the first oil shock, to the low inflation observed nowadays in a majority of OECD countries.

We expect to observe periods of fairly stable inflation corresponding to periods when the inflation expectations of the public is equal to the actual inflation rate. We further assume that changes from one state to another happen smoothly over time. These regimes are distorted by short to medium term phenomena such as the business cycle, or stringent policymaker decisions. In this context we perform a descriptive decomposition of the inflation time series $\pi_{t}$ into three components of variation: a low frequency component 
$\rho_{t}$ (regime-trend) indicating the general level of inflation in the economy at a particular time $t$; a medium frequency component $\gamma_{t}$ (cycle-trend) explaining medium to short term variations either cyclical or transient and a high frequency component $\eta_{t}$ dealing with isolated shocks and other irregularities. This can be written

$$
\pi_{t}=\rho_{t}+\gamma_{t}+\eta_{t}
$$

The various components in (2) are extracted by a two-step procedure. The first step uses a filter catching low frequencies to obtain the general trend, $\rho_{t}$, which is then subtracted from the original series. In the second step, a higher-pass frequency filter is used to extract the cycle-trend component $\gamma_{t}$ that, once subtracted from the series, leaves us with the short term component $\eta_{t}$. In both steps the filtering of the components is done with locally weighted polynomial regression or loess (see Cleveland, Devlin and Grosse, 1988; and Cleveland et al., 1990).

We will now briefly describe the loess regression method used to compute $\rho_{t}$. Consider $\pi_{t}$ as the dependent variable, and the time $t$ as the independent variable. The trend component at time $t=\tau, \rho_{\tau}$, is then obtained by locally regressing $\pi_{t}$ against $t$ as follows. A positive integer $h$ is chosen. The values of $h$ closest to $\pi_{\tau}$ are then given weights based on their time distance from $\tau$. Let $\lambda_{h}(\tau)$ be the $h$ th farthest time distance from $\tau$. The commonly used weighting function is

$$
W(x)=\left\{\begin{array}{cl}
\left(1-x^{3}\right)^{3} & \text { for } 0 \leq x<1 \\
0 & \text { for } x \geq 1
\end{array}\right.
$$


The weight for any $\pi_{t}$ is then $\omega_{t}(\tau)=W\left(|t-\tau| / \lambda_{h}(\tau)\right)$. Thus, weights decrease as we get further away from the time $\tau$ and become zero at $h$ th time distance. The next step is to fit a polynomial of degree $d$ to the data with weight $\omega_{t}(\tau)$ at $\left(t, \pi_{t}\right)$. The value of the polynomial fit at time $\tau$ yields $\rho_{\tau}$. This procedure is repeated for every time point, $\tau$, at which we observed the inflation. Finally, $\gamma_{t}$ is filtered identically once $\rho_{t}$ is subtracted from $\pi_{t}$, although different values for the tuning parameters $h$ and $d$ are used.

The decomposition (2) performed by the above procedure is not unique and depends on the values for $h$ and $d$ that are chosen in both steps of the filtering procedure. As $h$ increases the regression curve becomes smoother, that is lower frequency variations are captured. Increasing $d$ enables more flexibility in curvature changes, which is convenient when high frequency components are extracted.

The above procedure is used to decompose the inflation time series for 23 OECD-countries. As an illustration, the decomposition for the United Kingdom is graphically displayed in Figure 1. The tuning parameters used are $h=35$ and $d=1$ to extract $\rho_{t}$, while $h=15$ and $d=2$ are used for $\gamma_{t}$. By inspecting the function $w_{t}(\tau)$, we can note that $h=35$ corresponds to regressing locally in a window of eight to nine years and $h=15$ in a window of four years. These tuning parameters values were chosen such that the long term trend would not be too sensitive to business cycles, whose variation is expected to be cached by $\gamma_{t}$. Note that the decompositions obtained are not very sensitive to small changes in $h$. Choosing very different values for these parameters may yield a decomposition of the inflation that is still interesting, but for other purposes than those of this paper. Finally, note that we have 
used identical tuning parameters for all the inflation time series. We believe this to be important for the decompositions to be comparable.

\section{Central bank independence and price stability}

In this section, the long-term component, $\rho_{t}$, is compared with the implementation dates of central bank independence reforms in order to study the impact of central bank independence on price stability. We define that price stability as having been achieved when the long-term trend component, $\rho_{t}$, lies below three percent. $^{2}$ Announcement and implementation dates of central bank independence reforms are displayed in Table 1 for 23 OECD countries. ${ }^{3}$ Note that price stability, according to the time inconsistency theory, cannot be explained by a pre-commitment to central bank autonomy. If announcement of a central bank independence reform alters the inflation expectations of the public, then it is optimal for the policymakers to violate this promise when price stability is achieved (for a similar argument, see McCallum, 1997).

We consider reforms that alter the institutional framework for the central bank as independence reforms. Thus, a statement that price stability is the only goal for the monetary policy is not regarded as sufficient. According to our definition, the price stability target must be safeguarded in the leg-

\footnotetext{
${ }^{2}$ Madheva and Sterne (2000) defines price stability as a period when inflation remains in a specific range for a five-year period, and the lowest inflation range is defined as inflation below 3.8 percent. This means that our definition is stronger. Note, however, that the low inflation goal for the ECB is two percent.

${ }^{3}$ Announcement and implementation dates have been obtained from official documents (e.g., central bank acts,), scientific articles, and e-mail correspondence with researchers at universities and central banks.
} 
islation. However, several studies (see e.g., Svensson, 1997; Mishkin, 1999; Johnson, 2002; Neuman and von Hagen, 2002) have argued that inflation targeting can be used to solve the time inconsistency problem in monetary policy. The long-term component, $\rho_{t}$, is, therefore, also compared with the beginning of inflation targeting to check whether the more extensive use of explicit inflation targets can explain the transition from high to low inflation. The starting dates of inflation targeting are obtained from Ball and Sheridan's (2003) work, which means that the beginning of inflation targeting is defined as "the first full quarter in which a specific inflation target or target range was in effect, and the target had been announced publicly at some earlier time" (Ball and Sheridan, 2003 p. 5).

As can be seen from Table 1, 17 of the 23 OECD-countries studied in this paper have implemented institutional reforms granting their central banks more independence from the political policymakers. For the members of the ESCB (European System of Central Banks), this may be explained by the outline of the Maastricht Treaty where their national central banks were required to be independent before the ESCB's establishment date. ${ }^{4}$

The long-term components, the beginning of inflation targeting, and the implementation dates of central bank independence reforms are graphically displayed in Figures 2, 3, 4 and 5. These displays indicate that price stability has been achieved before the implementation date in a majority $(11 / 17)$ of the countries that have undertaken central bank independence reforms.

\footnotetext{
${ }^{4}$ The Maastricht Treaty on European Economic and Monetary Union was announced on 9-10 December 1991, and implemented on 1 November 1993. For a prospective member of the European Union, the convergence criteria stipulate that inflation should not exceed the average inflation in the three EU-countries with the lowest inflation by more than 1.5 percentage. ESCB was established on 1 January 1999.
} 
Central bank independence cannot, therefore, explain the observed shift from high to low inflation. Price stability has also been achieved in Canada, Denmark, Germany, Norway and the USA, countries where no independence reforms have been implemented.

Price stability was reached only after the implementation of the central bank reform in Greece, New Zealand, Italy, Portugal and Spain. However, the implementation dates were very close to the price stabilization dates in these cases, indicating that the transition from high to low inflation was almost finished when the central bank independence reforms were implemented.

For some countries (e.g., Ireland and the Netherlands) the long-term trend inflation component rises above the three percent level at the end of the period studied. However, the decomposition at the end points of the considered time periods are less reliable due to the lack of data in one time direction. ${ }^{5}$ The period of higher inflation may, thus, be a short-term phenomenon even if it is apparent in the long term component.

Turning to the effect of inflation targeting, the results show that six of nine countries achieved price stability before they adopted inflation targeting. The exceptions are Spain, United Kingdom and New Zealand, but the transition from high to low inflation was in those cases almost finished when inflation targeting was imposed. This is in accordance with the evidence presented by Ball and Sheridan (2003), who found no indication that inflation targeting improved economic performance as measured by the behavior of inflation.

\footnotetext{
${ }^{5}$ This is known as the end effect problem in the non-parametric regression literature.
} 
To summarize, it does not seem to be necessary to restrict the decision power of the policymakers, as suggested by for example, Rogoff (1985), Persson and Tabellini (1993), and Walsh (1995), in order to achieve credibility for a low inflation rule. The observed transition from high to low inflation cannot either be explained by the more extensive use of inflation targeting during the 1990s. On the other hand, more independence for the central banks and inflation targeting may have played an important role in stabilizing the inflation rates at a low level.

\section{Conclusions}

In this study we could not find evidence for one of the most influential empirical findings in macro-economics of the last decade, namely the negative relationship between the degree of independence of the central bank and the inflation rate. By comparing the implementation dates of central bank independence reform with the long term inflation trends for 23 OECD-countries, we were not able to find any signs to suggest that more central bank independence has contributed to lower inflation rates and price stability. On the contrary, price stability seems to have been achieved in most countries before their central banks did became more independent. This may indicate that central bank independence reforms have been used as an instrument against future uncarefull policymakers. Moreover, low and stable inflation has been reached in countries that have chosen not to change the legislation concerning the status of their central banks. The more extensive use of inflation targeting during the 1990s could not either explain the observed 
transition from high to low inflation.

These findings suggest that price stability have been achieved by other means than central bank independence and inflation targeting. The shift from high to low inflation may, for example, have been caused by exchange rate aggreements, the more extensive use of price stability targets (see e.g., Table 3.1 in Madhava and Sterne, 2000) or the announcment in the Maastrich Treaty that the ECB will be the central bank in the future. However, according to the time inconsistency theory in monetary policy, these arrangments are not sufficient because they can be violated when the low inflation goal is achieved. The time inconsistency theory clearly suggests that the decision power of the policymakers must be reduced in order to improve the credibility of a low inflation goal. However, this policy implication depends crucially on the assumption that the policymakers unemployment target is lower than the natural rate of unemployment. The periods of high inflation can have led the policymakers to become more inflation averse and the transition from high to low inflation may, therefore, be explained by shifting preferences. Hence, central bank autonomy can have been institutionalized at a time when the policymakers have a commitment to low inflation and used as a commiting device against subsequent governments.

While the increase in degree of central bank independence or the use of inflation targeting could not be correlated to a shift from high to low inflation (defined as long term inflation trend below three percent), this study cannot reject the idea that low inflation cannot be guaranteed in the long run with independent central banks or inflation targeting. Because most of the central bank and inflation targeting reforms took place in the 
nineties, more time is needed before such an hypothesis may be empirically studied. 


\section{References}

Agell, J., Calmfors, L., Jonsson, G., 1996. Fiscal policy when monetary is tied to the mast. European Economic Review 40, 1413-1440.

Alesina, A., Summers, L., 1993. Central bank independence and macroeconomic performance: some comparative evidence. Journal of Money, Credit and Banking 25, 151-162.

Ball, L., Sheridan, N., 2003. Does inflation targeting matter? NBER Working Paper 9577.

Barro, R.J., Gordon, D.B., 1983. A positive theory of monetary policy in a natural rate model. Journal of Political Economy 91, 101-121.

Blake, A.P., Weale, M., 1998. Costs of separating budgetary policy from control of inflation: a neglected aspect of central bank independence. Oxford Economic Papers 50, 449-467

Cleveland, R.B., Cleveland, W.S., McRae, J.E., Tevpenning, I., 1990. STL: a seasonal-trend decomposition procedure based on Loess. Journal of Official Statistics 6, 3-73.

Cleveland, W.S., Devlin, S.J., Grosse, E., 1988. Regression by local fitting: methods, properties, and computational algorithms. Journal of Econometrics $37,87-114$.

Cukierman, A., Webb, S.B., Neyapti, B., 1992. Measuring the independence of central banks and its effects on policy outcomes. World Bank Economic Review 6, 353-398.

Eijffinger, S., Schaling, E., Hoeberichts, M., 1998. Central bank indepen- 
dence: a sensitivity analysis. European Journal of Political Economy 14, 73-88.

Forder, J., 1996. On the assessment and implementation of institutional remedies. Oxford Economic Papers 48, 39-51.

Forder, J., 1998a. Central bank independence: conceptual clarifications and interim assesment. Oxford Economic Papers 50, 307-334.

Forder, J., 1998b. The case for an independent European central bank: a reassessment of evidence and sources. European Journal of Political Economy 14, 53-71.

Grilli, V., Masciandaro, D., Tabellini, G., 1991. Political and monetary institutions and public financial policies in the industrial countries. Economic Policy 6, 341-392.

Hayo, B., 1998. Inflation culture, central bank independence and price stability. European Journal of Political Economy 14, 241-263.

Hayo, B., Hefeker, C., 2002. Reconsidering central bank independence. European Journal of Political Economy 18, 653-674.

Johnson, D.R., 2002. The effect of inflation targeting on the behavior of expected inflation: evidence from an 11 country panel. Journal of Monetary Economics 49, 1521-1538.

Jonsson, G., 1995. Institutions and macroeconomic outcomes - the empirical evidence. Swedish Economic Policy Review 2, 181-212.

Kydland, F.E., Prescott, E.C., 1977. Rules rather than discretion: the inconsistency of optimal plans. Journal of Political Economy 85, 473 - 490. 
Mahadeva, L., Sterne, G., 2000. Monetary policy frameworks in a global context. Routledge, London.

Mangano, G., 1998. Measuring central bank independence: a tale of subjectivity and of its consequences. Oxford Economic Papers 50, 468-492.

McCallum, B.T., 1997. Crucial issues concerning central bank independence. Journal of Monetary Economics 39, 99-113.

Mishkin, F.S., 1999. International experience with different monetary policy regimes. Journal of Monetary Economics 43, 579-605.

Neuman, M.J.M., von Hagen, J., 2002. Does inflation targeting matter?. Federal Reserve Bank of St. Louis Review 84, 127-148.

Persson, T., Tabellini, G., 1993. Designing institutions for monetary stability. Carnegie-Rochester Conference Series on Public Policy 39, 53-84.

Posen, A., 1993. Why central bank independence does not cause low inflation: there is no institutional fix for politics. In: O'Brien, R. (ed.), Finance and the international economy 7. Oxford University Press, Oxford.

Posen, A., 1998. Central bank independence and disinflationary credibility: a missing link?. Oxford Economic Papers 50, 335-359.

Rogoff, K., 1985. The optimal degree of commitment to an intermediate monetary target. Quarterly Journal of Economics 100, 1169-1190.

Svensson, L.E.O., 1997. Inflation forecasting targeting: implementing and monitoring inflation targets. European Economic Review 41, 1111-1146.

Walsh, C., 1995. Optimal contracts for central bankers. American Economic 
Review 85, 150-167. 


\section{Decomposition of the UK inflation}

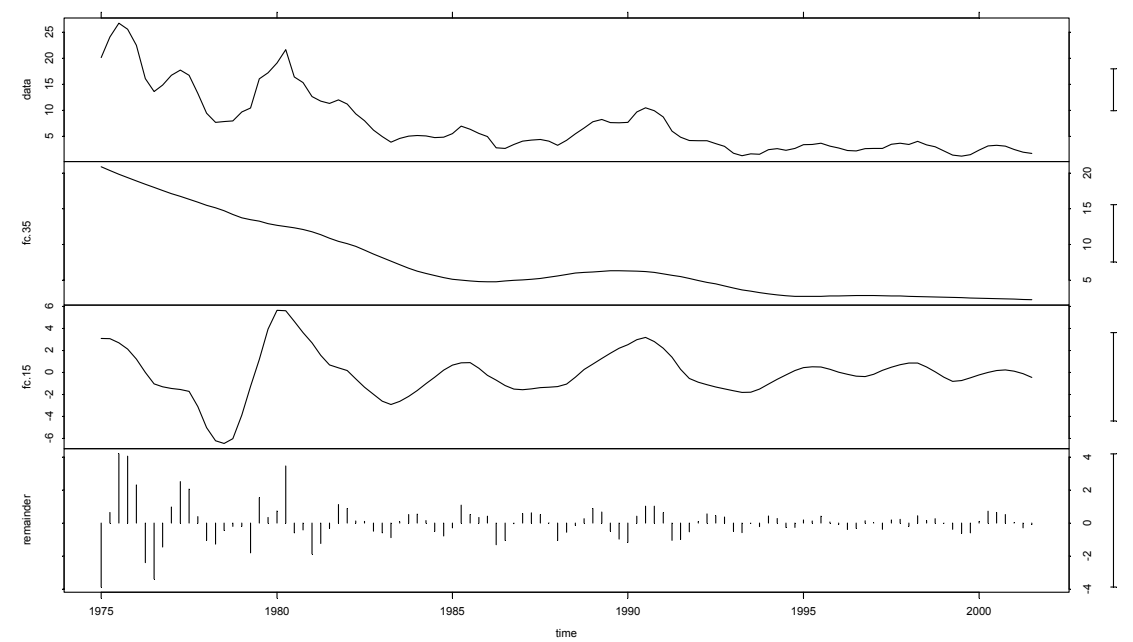

Figure 1: From top to bottom, the UK inflation time series $\pi_{t}$, the general trend $\rho_{t}$, the cycle-trend $\gamma_{t}$ and the short term component $\eta_{t}$ are displayed. Thus, for each time point $t$, the upper graph is the sum of the three others. The vertical lines on the right hand side of the graphs allows for comparison of the different scales. 
Table 1: Central bank independence reforms.

\begin{tabular}{lcc}
\hline \hline Countries & Announcement date & Implementation date \\
\hline Austria & $1998-04-24$ & $1999-01-01$ \\
Australia & None & None \\
Belgium & $1993-03-22$ & $1993-04-19$ \\
Canada & None & None \\
Denmark & None & None \\
Finland & $1997-07-24$ & $1998-01-01$ \\
France & $1993-08-04$ & $1994-01-01$ \\
Greece & $1997-11-* *$ & $1997-12-22$ \\
Germany & None & None \\
Japan & $1997-06-11$ & $1998-06-22$ \\
Iceland & $2001-03-27$ & $2001-05-27$ \\
Ireland & $1998-05-02$ & $1998-06-01$ \\
Italy & $1998-03-14$ & $1998-04-* *$ \\
Luxemburg & $1998-12-23$ & $1999-01-01$ \\
Netherland & $1998-05-02$ & $1998-06-01$ \\
New Zealand & $1989-12-15$ & $1990-02-01$ \\
Norway & None & None \\
Portugal & $a$ & $1998-01-31$ \\
Spain & $1994-06-01$ & $1994-06-02$ \\
Sweden & $1998-03-04$ & $1999-01-01$ \\
Switzerland & $1999-04-18$ & $2000-01-01$ \\
United Kingdom & $1997-05-06$ & $1998-06-01$ \\
USA & None & None \\
\hline
\end{tabular}


Canada

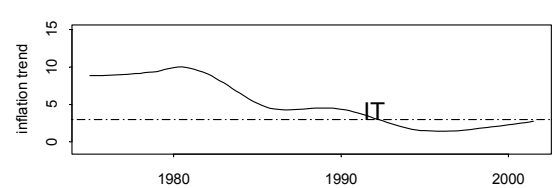

Denmark

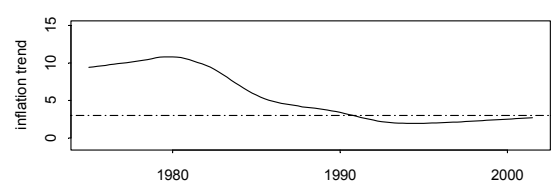

USA

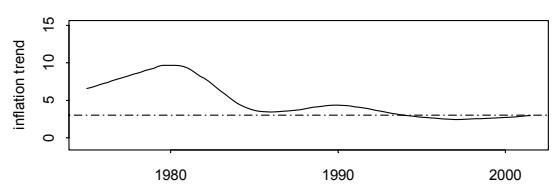

Australia

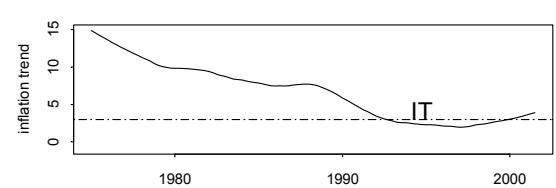

Norway

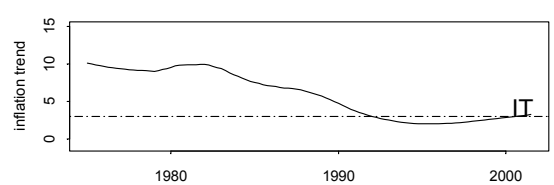

Germany

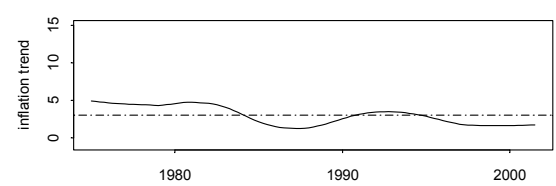

Figure 2: The general long trend component $\rho_{t}$, for six countries where no central bank reform was undertaken. The starting dates of inflation targeting are highlighted with the letters "IT". 

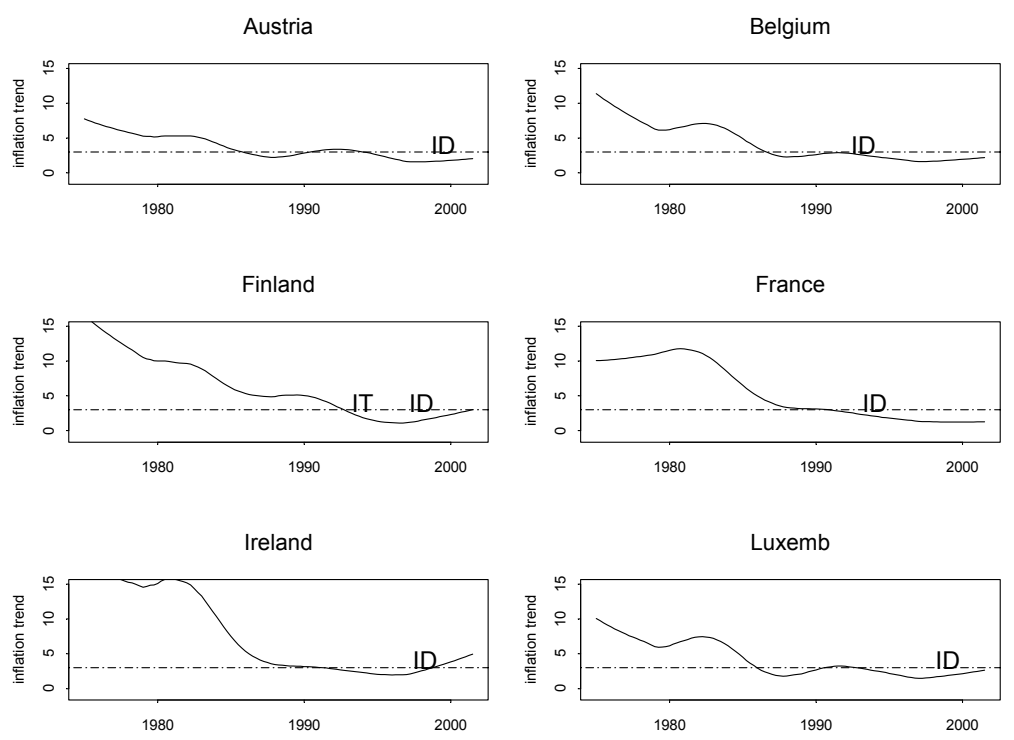

Figure 3: The general long trend component $\rho_{t}$, for six countries where a central bank reform was undertaken at time highlighted with the letters "ID", for implementation date; see Table 1. These countries, as those of Figure 4, have the particularity of reaching a low long trend inflation (below $3 \%$, dashed horizontal line) before the reform is implemented. The starting dates of inflation targeting are highlighted with the letters "IT". 

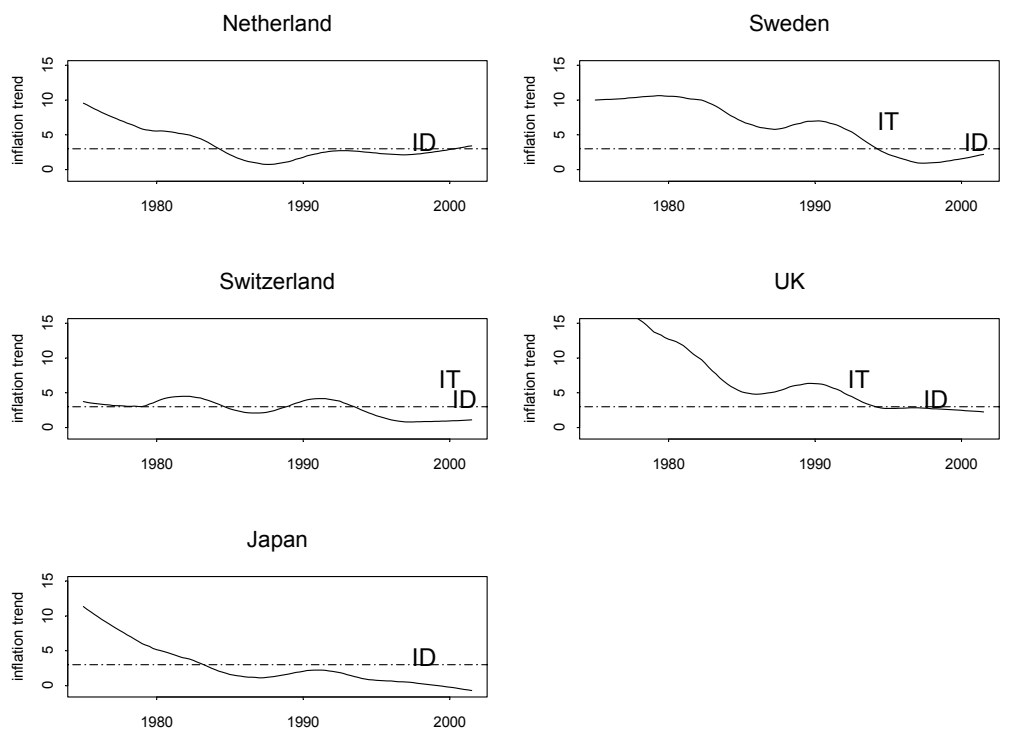

Figure 4: The general long trend component $\rho_{t}$, for five countries where a central bank reform was undertaken at time highlighted with the letters "ID", for implementation date; see Table 1. These countries, as those of Figure 3, have the particularity of reaching a low long trend inflation (below $3 \%$, dashed horizontal line) before the reform is implemented. The starting dates of inflation targeting are highlighted with the letters "IT". 

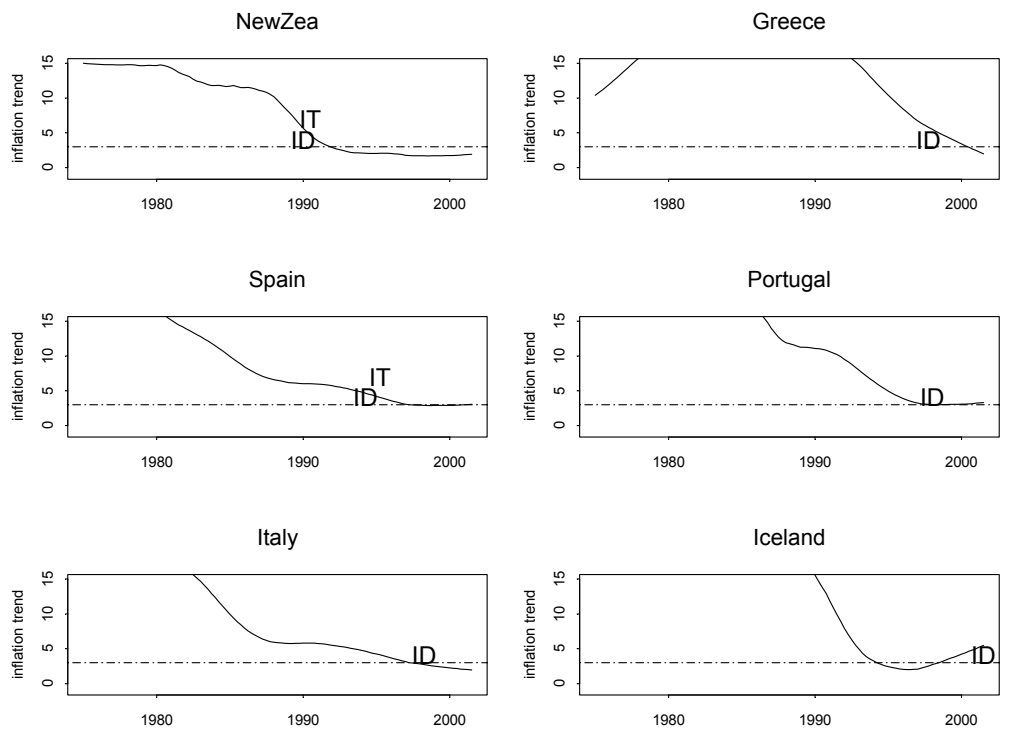

Figure 5: The general long trend component $\rho_{t}$, for six countries where a central bank reform was undertaken at time highlighted with the letters "ID", for implementation date; see Table 1 . These countries have the particularity of not reaching a low long trend inflation (below $3 \%$, dashed horizontal line) before the reform is implemented. The starting dates of inflation targeting are highlighted with the letters "IT". 